\title{
Submental and Diaphragmatic Muscle Activity during and at Resolution of Mixed and Obstructive Apneas and Cardiorespiratory Arousal in Preterm Infants
}

\author{
HENNING WULBRAND, GEORG VON ZEZSCHWITZ, AND KARL H. P. BENTELE
}

University of Hamburg, Department of Pediatrics, Germany

ABSTRAC'T

\begin{abstract}
Pathomechanisms involved in obstructive apneas remain obscure. Apnea arousal failure has been proposed as a cause for sudden death during sleep. The present study hypothesizes an interdependency between upper airway dilating submental muscle electromyogram (EMG) activity (EMGsub), diaphragmatic muscle activity (EMGdia), incidence of bradycardia, and transcutaneous measured $\mathrm{PO}_{2}\left(t c p \mathrm{O}_{2}\right)$ upon termination of apnea. Polygraphic recordings, including surface EMG (EMGsub, EMGdia), EEG, ECG, and transcutaneous $\mathrm{PO}_{2} / \mathrm{PCO}_{2}\left(\right.$ tcpO $\mathrm{O}_{2} /$ tcp $\mathrm{CO}_{2}$ ) were performed on 10 preterm infants at $36,40,44$, and $52 \mathrm{wk}$ of conceptional age. EMGsub increased initially, then decreased in 28 of 33 non-rapid eye movement (N-REM) sleep apneas (REM: 35 of 69 events). This correlated with a decrease of tcpO2 during N-REM sleep $(p<0.05)$. A parallel decrease of EMGsub and EMGdia was correlated with the occurrence of bradycardia (REM and N-REM: $p<0.01$ ). Concomitant termination of apnea and bradycardia $(n=22)$, occurred in the presence of a phasic, simultaneous activation of EMGsub and EMGdia in $64 \%$ of REM sleep and in $79 \%$ of N-REM sleeprelated event, was characterized by a deep inspiration preceded by a short expiration, and correlated with the extent of tcpO ${ }_{2}$
\end{abstract}

Prolonged apneas accompanied by hypoxemia frequently occur in preterm infants (1-3) especially in those recovering from respiratory distress syndrome (4) during the first months of life. A loss of balance between submental EMG activity (representing mainly the protruding and thus upper airway dilating muscle force of $\mathrm{m}$. genioglossus) and diaphragmatic EMG has been proposed as playing a leading role in the incidence of obstructive apneas (5-7). In contrast to adults (8), in children no significant decrease of EMGsub was found at onset of apneas (9), but a preferential activation of EMGsub accompanied termination of apneas in both adults and children $(8,9)$. Bradycardia during apnea is a common finding (10).

Received February 17, 1994; accepted March 23, 1995.

Correspondence: Dr. Henning Wulbrand, University of Hamburg, Department of Pediatrics, Martinistr. 52, D-20251 Hamburg, Germany.

Supported by the Deutsche Forschungsgemeinschaft WU 171 1-1. decline during REM sleep apneas $(p<0.05)$. In one apnea with bradycardia that progressed to asystolia, this mechanism was missing, but was evoked by a slight tactile stimulation, whereupon cardiorespiratory functions were immediately reestablished whereas N-REM sleep continued uninterrupted. Our data demonstrate an interdependency between changes of EMGsub and EMGdia activity, $\mathrm{tcpO}_{2}$ decline, and occurrence of bradycardia. A "cardiorespiratory arousal" terminated apneas and bradycardia without a change in sleep phase. (Pediatr Res 38: 298-305, 1995)
Abbreviations
EMG, electromyogram
EMGsub, submental electromyogram
EMGdia, diaphragmatic electromyogram
REM, rapid eye movement
N-REM, non-rapid eye movement
tcpO $\mathrm{O}_{2}$, transcutaneous $\mathrm{PO}_{2}$
tcpCO $\mathrm{CO}_{2}$, transcutaneous $\mathrm{PCO}_{2}$
SIDS, sudden infant death syndrome

Even cessation of heartbeat during an apnea has been reported in one preterm infant (11). but underlying mechanisms regarding the origin and termination of apnea are still obscure. Although controversial, there is evidence that apneas may play an important role in SIDS $(12,13)$. In infants surviving an apparent life-threatening event a diminished hypoxic ventilatory arousal response could be demonstrated (14), which possibly has an influence on termination of apneas. Autoresuscitation by gasping is a well studied phenomenon in animals (15-17). Failure of this gasping mechanism in terminating apneas has been hypothesized as being the final and most devastating physiologic factor in SIDS victims (18). Preterm infants recovering from respiratory distress syndrome and bronchopulmonary dysplasia frequently suffer from apneas with an obstructive component (4), show an impaired chemoreceptor response to hypoxia (19), and are at increased risk of 
death during sleep (20). To reevaluate mechanisms initiating obstructive apneas as well as those related to cardiorespiratory control, such infants were investigated by means of polygraphic recordings including submental and diaphragmatic muscle activity, EEG, nasal and abdominal breathing activity, and tcpO $\mathrm{O}_{2}$ levels during apnea.

\section{METHODS}

Subjects. Ten preterm infants at conceptional age of 36,40 , 44, and 52 wk were studied (Table 1). Analysis of data from infants still being ventilated at conceptional age of 36 and 40 wk was excluded. Data from three infants were excluded at the conceptional age of $52 \mathrm{wk}$ due to pneumonia and bacterial meningitis. Gestational age ranged from 26.1 to $36.1(\chi=$ 29.4) wk, birth weight from 740 to $2020(\chi=1029.5) \mathrm{g}$, and ventilation duration between 2.2 and $16.3(\chi=15.2) \mathrm{wk}$. The neonatal risk factors included low birth weight, perinatal asphyxia, bacterial infections, transient periventricular echodensities, patent ductus arteriosus, and respiratory distress syndrome which in some cases had progressed to severe bronchopulmonary dysplasia. None of the infants suffered neurologic complications such as seizures or intracranial hemorrhage, which were ruled out by ultrasound examination. A bethamethasone prophylaxis was performed in all cases before delivery. No infant received theophylline within $7 \mathrm{~d}$ before recording. The study was conducted in accordance with the guidelines proposed in the "Declaration of Helsinki" and was accepted by the the Ethics Committee of the Chamber of Physicians, Hamburg, Germany. Informed consent was obtained from the parents, one of whom was present during the recordings.

Measurements. The polygraphic recordings included EEG (C4-P4, C3-P3), ECG (8-channel recorder, Schwarzer Electroencephaloscript 8000 Munich, Germany), recording of abdominal and thoracic movements measured by impedance (Hewlett/Packard HP 78201B Boiblinger, Germany), and tcpO $\mathrm{O}_{2}$ and $\mathrm{tcpCO} \mathrm{CO}_{2}$ (Hellige Transoxode Type 1234, Transkapnode Type 4567 Hamburg, Germany). Submental EMG was obtained by surface electrodes placed between the chin and hyoid bone. The diaphragmatic EMG was obtained by surface electrodes positioned at the right chest wall, subcostal and

Table 1. Patient information

\begin{tabular}{lccccc}
\hline & $\begin{array}{c}\text { Gestational } \\
\text { Patient } \\
\text { age (wk) }\end{array}$ & $\begin{array}{c}\text { Birthweight } \\
(\mathrm{g})\end{array}$ & $\begin{array}{c}\text { APGAR } \\
\text { at } 1 \text { min }\end{array}$ & $\begin{array}{c}\text { Duration of } \\
\text { ventilation } \\
(\mathrm{d})\end{array}$ & Neonatal risks* \\
\hline a & 27 & 740 & 8 & 61 & $1,2,3,4$ \\
b & 30 & 1390 & 9 & 23 & 2,4 \\
c & 30 & 940 & 8 & 53 & $1,3,4$ \\
d & 28 & 990 & 4 & 19 & 3,4 \\
e & 30 & 1200 & 6 & 36 & 3 \\
f & 32 & 1220 & 6 & 55 & 1,3 \\
g & 28 & 880 & 7 & 25 & 3,4 \\
h & 29 & 890 & 5 & 17 & 3,4 \\
i & 31 & 1035 & 9 & 19 & \\
k & 29 & 1010 & 8 & 44 & 1,4 \\
Mean & 29.4 & 1029.5 & 7 & 35.2 & \\
\hline
\end{tabular}

* Neonatal risks: 1, bronchopulmonary dysplasia III; 2, patent ductus arteriosus; 3 , transient periventricular echodensities; and 4 , proved septicaemia. pararectal in the medial clavicular line (Biometer 2000 Copenhagen, Denmark). Nasal airflow was measured by a thermistor placed below both nostrils. Recordings were performed during the day until conceptional age of $52 \mathrm{wk}$, after which recordings were obtained in the early evening. The recordings encompassed 5 to $6 \mathrm{~h}$ during sleep. The sleep stages were defined according to established criteria as described elsewhere (1, 21-23). Arousal was defined as a sudden change of the predominating pattern of EEG during sleep or in waking up (23-26). Bradycardia was defined as a deceleration of heart rate below 100 beats/min. Apneas were differentiated into "inactive," "obstructive," and "mixed" types. Inactive apneas were defined as periods of cessation of diaphragm inspiratory activity and abdominal and thoracal breathing movements as well as nasal airflow. In obstructive apneas breathing movements as well as inspiratory diaphragmatic EMG activity continued whereas nasal airflow was missing. Mixed apneas could show both types during the same apnea period with the inactive part preceding the obstructive part in all cases except one. EMG were recorded from silver/silver chloride surface electrodes filled with gel (Beckmann). Electrodes were attached to the skin using collodion. EMG activity was amplified a thousandfold using 20 and $3000 \mathrm{~Hz}$ high pass and low pass filters. The signals were rectified and integrated after digitalizing with a sampling rate of $8 \mathrm{kHz}$ (CED $1401 \mathrm{AD}$ converter Cambridge, UK).

Data analysis. To evaluate the changes of submental and diaphragmatic muscle activity at the onset, during, and at resolution, apneas were subdivided into thres sequences, each containing two windows of $1000 \mathrm{~ms}$ duration (Fig. 1), as follows. In sequence $a$, for analysis of EMGsub activity at onset of apneas window 1 beginning $4 \mathrm{~s}$ before apnea onset was compared with window 2 just at onset. In sequence $b$, for analysis of EMGsub during apneas window 3 beginning $2 \mathrm{~s}$ after apnea onset was compared with window 4 at $2 \mathrm{~s}$ before resolution apnea. In sequence $c$, for analysis of EMGsub activity at termination of apneas window 4 beginning $2 \mathrm{~s}$ before resolution of apnea was compared with window 5 just at resolution of apnea. In sequence $d$, for analysis of EMGdia activity during apneas window 6 at $4 \mathrm{~s}$ before apnea onset was compared with window 7 at $2 \mathrm{~s}$ before resolution of apnea. In sequence $e$, for analysis of EMGdia at termination of apnea window 7 at $2 \mathrm{~s}$ before resolution of apnea was compared with window 8 at resolution of apnea.

Because no inspiratory diaphragmatic EMG activity could be recorded for mixed apneas with an initial inactive part, these first windows had to be excluded from analysis. Only one inspiratory diaphragmatic EMG burst within each window was included in the analysis. Percentage of changes in submental and diaphragmatic EMG activity were correlated with the decrease of $t c \mathrm{O}_{2}(\mathrm{~mm} \mathrm{Hg})$ as well as the increase of tcpCO during apneas. Artifacts due to ECG were eliminated before analysis. An epoch of $20 \mathrm{~ms}$ before and after the R-wave was removed from the raw EMG signal and replaced by the mean activity of the adjoining EMG according to a method used elsewhere (27). Abdominal muscle activity due to body movements or expiration may confound diaphragmatic EMG measurements with its typical breathing synchronous form and was 


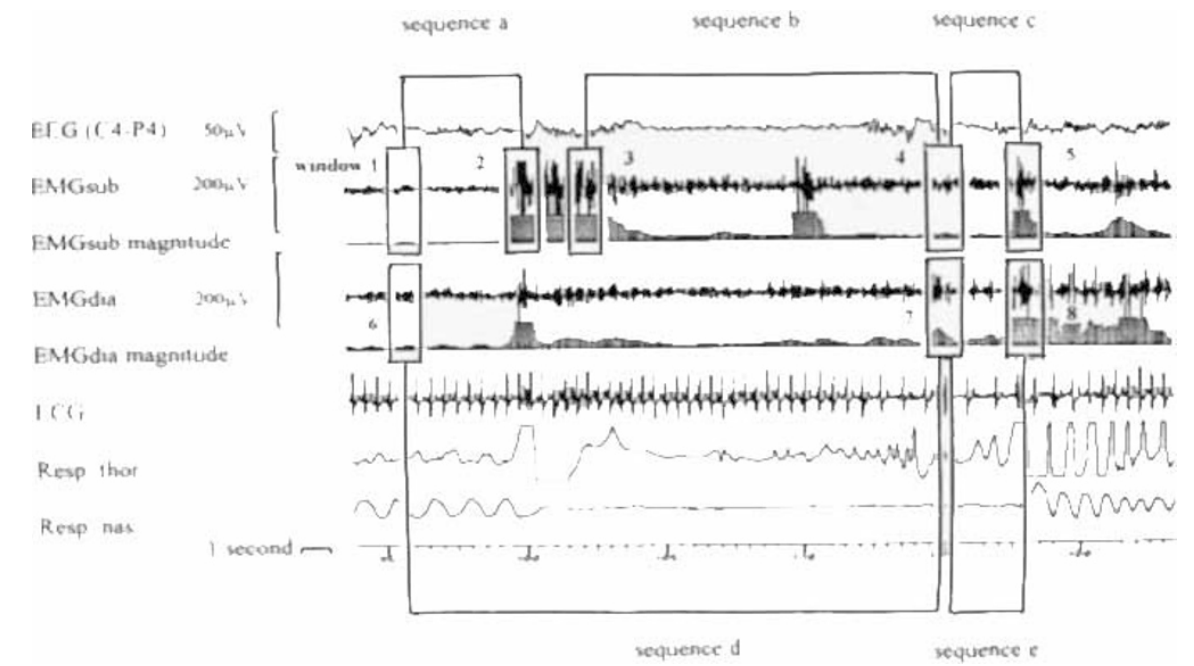

Figure 1. Recordings included EEG activity (C4-P4), submental (EMGsub) and diaphragmatic (EMGdia) surface EMG, ECG, thoracic movements, and nasal airflow. Integrated submental EMG was analyzed by selecting three sequences representing onset, course, and resolution of apneas, each containing one pair of windows of 1-s duration: the first sequence (sequence $a$ ) with a window $4 \mathrm{~s}$ before (window $l$ ) and another window just at onset of apnea (window 2 ), the second sequence (sequence b) containing a window $2 \mathrm{~s}$ after onset (window 3) and a window $2 \mathrm{~s}$ before resolution of apnea (window 4), the third sequence (sequence c) with a window $2 \mathrm{~s}$ before resolution of apnea (window 4) and window 5 just at resolution of apnea. The sequences for analyzing the EMGdia are parallel to those of EMGsub, but consist of only two sequences (sequences $d$ and $e$ ) with three windows (windows 6, 7, and 8 ) because diaphragmatic inspiratory EMG activity was absent during the inactive part of mixed apneas of the "inactive-obstructive" type. For comparison of the EMG activity change within each sequence values of the first window represent $100 \%$

thus differentiable. Submental EMG activity may be influenced not only by inspiration but also through swallowing or moving. The latter could be ruled out by visual observation and recording of an actogram but was never seen during N-REM sleep in our study. Measurement of tcpO2 is influenced by skin thickness, sensor temperature, and state of peripheral perfusion and provides only a delayed and attenuated reflection of changes of blood gases with a sensitivity of approximately 85\%. Thus although the recorded changes might underestimate real values, they appropriately reflect the tendency of blood gas changes during apneas (28). To evaluate $t \mathrm{cpO}_{2}$ and $t c \mathrm{cO}_{2}$ changes, comparative levels at the beginning of apneas and, according to the responding time of that method, at $10 \mathrm{~s}$ after its resolution were included in the analysis. Onset of apnea was defined as the beginning of the last inspiratory effort before the event. The first change of the nasal airflow after apnea defined its resolution. At resolution of apneas a specific pattern of a phasic, simultaneous EMG activation of both submental and diaphragmatic muscle groups was found to mark relief from obstruction. Phasic activation was defined as an increase of more than $80 \%$ of the mean integrated activity. A simultaneous activation of both muscle groups was given, if the time deviation between maximum of amplitudes of submental and diaphragmatic EMG was less than $200 \mathrm{~ms}$. For statistical evaluation the $\chi^{2}$ test, Fisher's exact test, Spearman rank correlation, and Wilcoxon rank test were used. Due to the small number of apneas at different conceptional ages, all recorded apneas with an obstructive component were included in the study and were considered to reflect a homogeneous pathophysiologic phenomenon during the first months of life in preterm infants recovering from respiratory distress syndrome.

\section{RESULTS}

General findings. A total of 33 apneas in N-REM (12 obstructive and 21 mixed events) and 69 in REM sleep (30 obstructive and 39 mixed) were recorded (Table 2). Apneas were preceded by an initial sigh in 16 REM sleep and in 8 N-REM-related events. The mean duration of apneas was 11.0 $\mathrm{s}( \pm 1.4 \mathrm{SD})$ in REM and $13.2 \mathrm{~s}( \pm 3.8 \mathrm{SD})$ in N-REM sleep with a maximum of $28 \mathrm{~s}$ in $\mathrm{N}$-REM sleep. A decrease in upper airway dilating submental muscle activity during apneas was found during $\mathrm{N}$-REM sleep relative to the extent of $t \mathrm{ppO}_{2}$ decline. Upper airway maintenance was reachieved by a gasplike simultaneous activation of both EMGsub and EMGdia at termination of apneas. Moreover, incidence of bradycardia correlated with a combined decrease of EMGsub and EMGdia during apneas. Bradycardia always ceased at termination of apnea, in parallel with a gasp but without change in EEG activity. This defined a "cardiorespiratory arousal."

Submental muscle activity at onset and during apneas. The onset of apneas (Fig. 1, sequence a; Table 3) was marked by a significant increase of submental EMG activity in REM sleep in 59 of 69 cases $(85.5 \%$ ) and in N-REM sleep in 29 of 33 cases $(74.3 \%)$. The quantitative increase of integrated submental muscle activity amounted to a mean of $50.1 \%$ ( $\pm 42.9 \mathrm{SD})$ in REM sleep and $61.9 \%( \pm 49.7 \mathrm{SD})$ in N-REM sleep. The occurrence of increasing EMGsub activities was significant in REM sleep as well as in N-REM sleep (Wilcoxon rank test, $p<0.05$ ). In contrast to the initial increase of submental EMG at onset of most apneas, only a slight decrease with a mean of $16.1 \%$ ( $\pm 85.0 \mathrm{SD}$ ) accompanied the events (Fig. 1, sequence b; Table 3): in 35 of the REM sleep-related apneas (50.7\%) a decrease was found, 34 showed an increase. N-REM sleep, on 
Table 2. Total apneas recorded

\begin{tabular}{|c|c|c|c|c|c|c|c|c|c|c|c|c|c|c|c|c|c|c|c|c|c|c|c|c|c|}
\hline \multirow{2}{*}{\multicolumn{2}{|c|}{ 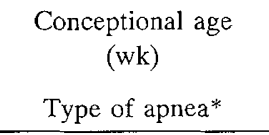 }} & \multicolumn{6}{|c|}{36} & \multicolumn{6}{|c|}{40} & \multicolumn{6}{|c|}{44} & \multicolumn{6}{|c|}{52} \\
\hline & & \multirow[t]{2}{*}{ ob. } & \multirow[t]{2}{*}{$\mathrm{g}$} & \multirow[t]{2}{*}{$\mathrm{b}$} & \multirow[t]{2}{*}{ mix. } & \multirow[t]{2}{*}{$\mathrm{g}$} & \multirow[t]{2}{*}{$\mathrm{b}$} & \multirow[t]{2}{*}{ ob. } & \multirow[t]{2}{*}{$\mathrm{g}$} & \multirow[t]{2}{*}{$\mathrm{b}$} & \multirow[t]{2}{*}{ mix. } & \multirow[t]{2}{*}{$\mathrm{g}$} & \multirow[t]{2}{*}{$b$} & \multirow[t]{2}{*}{$\mathrm{ob}}$. & \multirow[t]{2}{*}{$\mathrm{g}$} & \multirow[t]{2}{*}{$\mathrm{b}$} & \multirow[t]{2}{*}{ mix. } & \multirow[t]{2}{*}{$\mathrm{g}$} & \multirow[t]{2}{*}{$\mathrm{b}$} & \multirow[t]{2}{*}{ ob. } & $\mathrm{g}$ & $\mathrm{b}$ & $\operatorname{mix}$. & $g$ & $\mathrm{~b}$ \\
\hline Patients & Sleep phase & & & & & & & & & & & & & & & & & & & & & & & & \\
\hline $\mathrm{a}$ & N-REM & 1 & 1 & & 1 & 1 & 1 & 2 & 2 & & 2 & 2 & 2 & 1 & 1 & & 2 & 1 & 2 & & & & & & \\
\hline & REM & 2 & 1 & & 1 & 1 & & 3 & 2 & 1 & 3 & 2 & 1 & 1 & 1 & & 2 & 1 & & & & & 1 & & \\
\hline $\mathrm{b}$ & N-REM & & & & & & & 1 & 1 & 1 & 2 & 2 & 1 & & & & & & & & & & 2 & & \\
\hline & REM & 1 & 1 & & 2 & 1 & & 2 & 2 & & 2 & 1. & 1 & 2 & 2 & 1 & & & & & & & 1 & & \\
\hline c & N-REM & & & & & & & & & & 1 & 1 & & & & & & & & $-i$ & & & - & & \\
\hline & REM & 1 & 1 & & 1 & 1 & & 1 & 1 & & 2 & 1 & & 1 & 1 & & 1 & 1 & 1 & - & & & - & & \\
\hline $\mathrm{d}$ & N-REM & & & & 1 & & & 1 & 1 & 1 & 1 & & & & & & & & & - & & & - & & \\
\hline & REM & 2 & 1 & 1 & 2 & 2 & 1 & 1 & & & 1 & 1 & & 1 & 1 & & & & & - & & & - & & \\
\hline $\mathrm{e}$ & N-REM & & & & & & & & & & 1 & 1 & 1 & & & & 1 & & & & & & & & \\
\hline & REM & 1 & 1 & & 1 & & & 1 & & & 1 & 1 & 1 & 1 & & & 1 & 1 & & & & & & & \\
\hline $\mathrm{f}$ & N-REM & - & & & - & & & - & & & - & & & & & & & & & & & & & & \\
\hline & REM & - & & & - & & & - & & & - & & & 1 & & & 2 & 1 & & & & & & & \\
\hline $\mathrm{g}$ & N-REM & & & & 1 & 1 & 1 & & & & 1 & 1 & 1 & 1 & 1 & 1 & & & & & & & & & \\
\hline & REM & 2 & 1 & & 4 & 2 & & & & & 2 & 2 & 1 & & & & 1 & 1 & & & & & & & \\
\hline $\mathrm{h}$ & N-REM & & & & & & & & & & 3 & 2 & & 1 & & & & & & - & & & 一 & & \\
\hline & REM & 1 & 1 & & 2 & 1 & & 1 & 1 & & 1 & 1 & & & & & & & & - & & & 一 & & \\
\hline $\mathrm{i}$ & N-REM & 1 & 1 & 1 & & & & 1 & & & & & & & & & 1 & & & & & & & & \\
\hline & REM & 2 & 2 & & 1 & 1 & & & & & 1 & 1 & & 1 & 1 & & & & & & & & & & \\
\hline $\mathrm{k}$ & N-REM & - & & & - & & & 1 & 1 & & 1 & 1 & & & & & & & & 1 & & & & & \\
\hline & REM & - & & & - & & & & & & 1 & & & & & & 1 & & & & & & & & \\
\hline Sum: & N-REM & 2 & 2 & 1 & 3 & 2 & 2 & 6 & 5 & 2 & 12 & 10 & 5 & 3 & 2 & 1 & 4 & 3 & 2 & 1 & 1 & & 2 & 1 & \\
\hline & REM & 12 & 9 & 1 & 14 & 10 & 1 & 9 & 6 & 1 & 15 & 10 & 4 & 8 & 6 & 1 & 8 & 3 & 1 & 1 & & & 2 & & \\
\hline
\end{tabular}

* ob, obstructive apnea; $g$, apnea terminating gasp; b, bradycardia $(<100 / \mathrm{min})$; mix, mixed apnea.

$\dagger-=$ no record.

Table 3. Number of apneas with increasing or decreasing EMG activity (mean extent of EMG activity change: \% $\pm S D$ ) at onset and during apneas

\begin{tabular}{lrrr}
\multicolumn{4}{c}{ and during apneas } \\
\multicolumn{3}{c}{ Increase Decrease } & Extent \\
\hline REM & & & \\
EMGsub & 59 & 10 & $\chi=+50.1 \% \pm 42.9 \mathrm{SD} \dagger$ \\
$\quad$ At onset* & 34 & 35 & $\chi=-16.1 \% \pm 85.0 \mathrm{SD}$ \\
$\quad$ During apnea & 35 & 34 & $\chi=+27.0 \% \pm 64.2 \mathrm{SD}$ \\
EMGdia during apnea & & & \\
N-REM & & & \\
EMGsub & 29 & 4 & $\chi=+61.9 \% \pm 49.7 \mathrm{SD}$ \\
$\quad$ At onset* & 5 & 28 & $\chi=-38.8 \% \pm 39.9 \mathrm{SD}$ \\
$\quad$ During apnea* & 15 & 18 & $\chi=+9.1 \% \pm 48.5 \mathrm{SD}$ \\
EMGdia during apnea &
\end{tabular}

* Wilcoxon test: $p<0.05$.

$\uparrow$ Mean extent: $\% \pm \mathrm{SD}$.

the other hand, was characterized by a predominance of decreasing submental EMG activity of $38.8 \%$ ( $\pm 39.9 \mathrm{SD}): 28$ of 33 episodes ( $84.4 \%$ of the apneas) showed a decrease (Wilcoxon rank test, $p<0.05$ ). EMGdia (Table 3; Fig. 1, sequence d) increased by a mean of $27.0 \%( \pm 64.2 \mathrm{SD})$ in REM-related apneas with 35 of 69 apneas (50.7\%) showing increasing activity. During N-REM sleep EMGdia activity increased in 15 of 33 events $(45.5 \%$ ), with a mean increase of only $9.1 \%$ $( \pm 48.5 \mathrm{SD})$.

Submental and diaphragmatic EMG activity at resolution of apneas. At resolution of the apneas a phasic, simultaneous activation of submental and diaphragmatic EMG was observed. Especially remarkable was that the deep inspiration terminating the apneas was preceded by a short expiration (Fig. 2). This phasic, simultaneous EMG activation was found in 44
$(63.8 \%)$ of the REM sleep related apneas and in $26(78.8 \%)$ of the N-REM sleep-related apneas. The integrated activity of EMGsub increased in REM sleep by a mean of $129.7 \%( \pm 49.5$ SD) and in N-REM sleep by $117.3 \%$ ( \pm 38.5 SD), whereas EMGdia rose by $158.3 \%$ ( $\pm 60.5 \mathrm{SD})$ in REM sleep and $188.2 \%( \pm 77.3 \mathrm{SD})$ in N-REM sleep (Fig. 1, sequences $\mathrm{c}$ and e). The remaining apneas $(n=25,36.2 \%$ in REM sleep; $n=$ 7, $21.2 \%$ in N-REM sleep) showed either decreasing or increasing EMG activities of EMGsub and EMGdia, never reaching the synchronicity of activation as described above.

Submental and diaphragmatic EMG activity and tcp $\mathrm{O}_{2} /$ $\mathrm{CO}_{2}$ levels. Although EMGsub showed both decreasing and increasing EMG activities, a significant correlation was found between the degree of $\mathrm{cpO}_{2}$ decline and EMGsub and EMGdia: the greater the decrease in $t c \mathrm{O}_{2}$ level, the greater the decrease in EMGsub and EMGdia (Spearman rank correlation, $p<0.05$; Fig. 3) in N-REM sleep. No correlation was found during REM sleep (Fig. 4). Moreover, no correlation was found between tcpCO $\mathrm{CO}_{2}$ and augmented EMG activity during or at resolution of apneas. The appearance of phasic and simultaneous submental/diaphragmatic EMG activation in REM sleep showed a significant correlation with the degree of decrease of $t \mathrm{cpO}_{2}$ levels (Fisher's exact test, $p<0.01$, Table 4). In N-REM sleep, such a correlation was not found because this specific EMG pattern predominated, independent of the degree of $\mathrm{tcpO}_{2}$ decrease.

Parallel decrease of EMGsub and EMGdia activity during apneas and the appearance of bradycardia. A parallel decrease of EMGsub and EMGdia was found in REM sleep in 18 $(24.6 \%)$ and in N-REM sleep in 14 (42.2\%) apneas. Bradycar- 


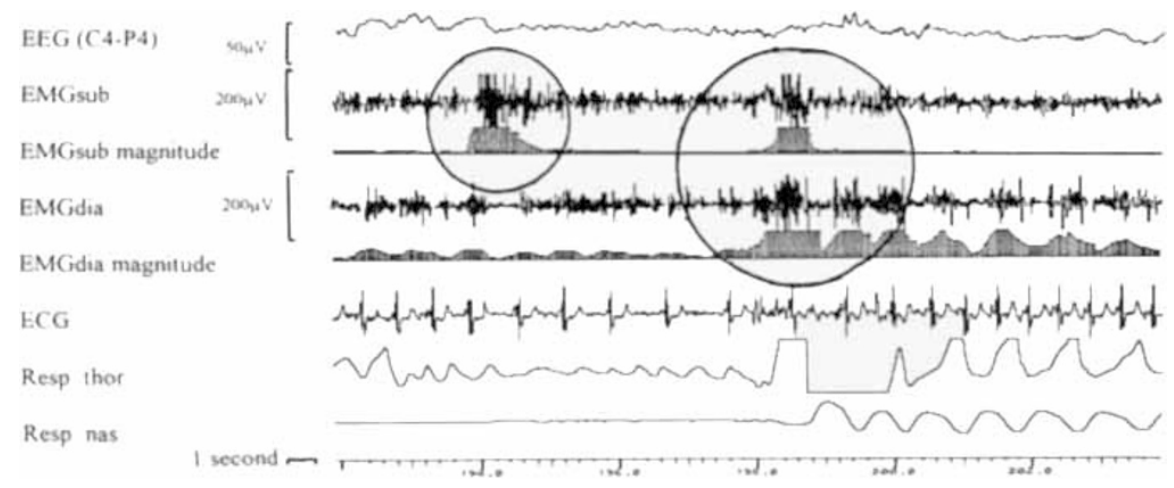

Figure 2. EMGsub and EMGdia during and at resolution of mixed and obstructive apneas. A mixed (inactive-obstructive) apnea with onset of thoracic and abdominal movements as well as diaphragmatic inspiratory activity displays an initial increase of EMGsub at onset, which subsequcntly decreases. Obstruction continues despite an isolated activation of submental EMG in the course of the event (small circle) until the parallel occurrence of a phasic, simultaneous EMG activation of submental and diaphragmatic EMG (large circle), which coincides with relief from obstruction and termination of bradycardia. The registration of diaphragmatic EMG shows the beginning of this activation at the end of the inspiratory phase of the last inspiratory effort against obstruction whereas nasal airflow starts again in accordance with an expiration before continuation of normal breathing

dia occurred in 9 cases during REM sleep and in 13 cases during N-REM sleep-related apneas. There was a significant predominance of bradycardia during apneas with parallel decreasing activities of EMGsub and EMGdia in REM sleep in 8 of $9\left(\chi^{2}: p<0.001\right.$; Table 5) and in N-REM sleep in 10 of 13 cases $\left(\chi^{2}: p<0.01\right.$; Table 5$)$. In all apneas, bradycardia began during the last seconds of the event. As in all but one of the mixed apneas, the obstructive activity was preceded by the inactivation, and bradycardia was always related to obstruction. With the appearance of the phasic simultaneous activation of EMGsub and EMGdia, not only termination of apnea but also of bradycardia occurred.

Occurence of bradycardria progressing to asystolia during one mixed apnea. During one of a number of mixed apneas of one particular infant (gestational age 30, conceptional age 40 wk) with decreasing activities of EMGsub and EMGdia, the characteristic simultaneous EMG pattern at apnea resolution was missing (Fig. 5). After onset of bradycardia during the obstructive part of the mixed event, heartbeat ceased only $13 \mathrm{~s}$ after apnea onset, whereas inspiratory efforts continued with decreasing amplitude. The $t c \mathrm{PO}_{2}$, already suppressed during preceding apnea episodes to $5.5 \mathrm{~Pa}$, further dropped to 5.1 $\mathrm{Pa}$. A slight tactile stimulation of the infant evoked the missed phasic, simultaneous EMG pattern and led to immediate reestablishment cardiorespiratory functions, whereas N-REM sleep continued uninterrupted.

\section{DISCUSSION}

Submental and diaphragmatic EMG at onset of apneas. This study demonstrates the predominance of decreasing submental EMG activity during apneas in N-REM sleep after an initial increase at onset of apneas with an obstructive component in preterm infants recovering from respiratory distress syndrome. It shows that the decrease of submental as well as diaphragmatic EMG activity during the course apneas is correlated with the decline of $t \mathrm{cpO}_{2}$ levels during $\mathrm{N}$-REM sleep and that termination of mixed and obstructive apneas is characterized by a gasp. This is in contrast to other findings in adults (8), children (29), and preterm infants (27). Whereas a decrease of genioglossus activity has been described in adults at onset of apneas, possibly leading to obstruction of the upper

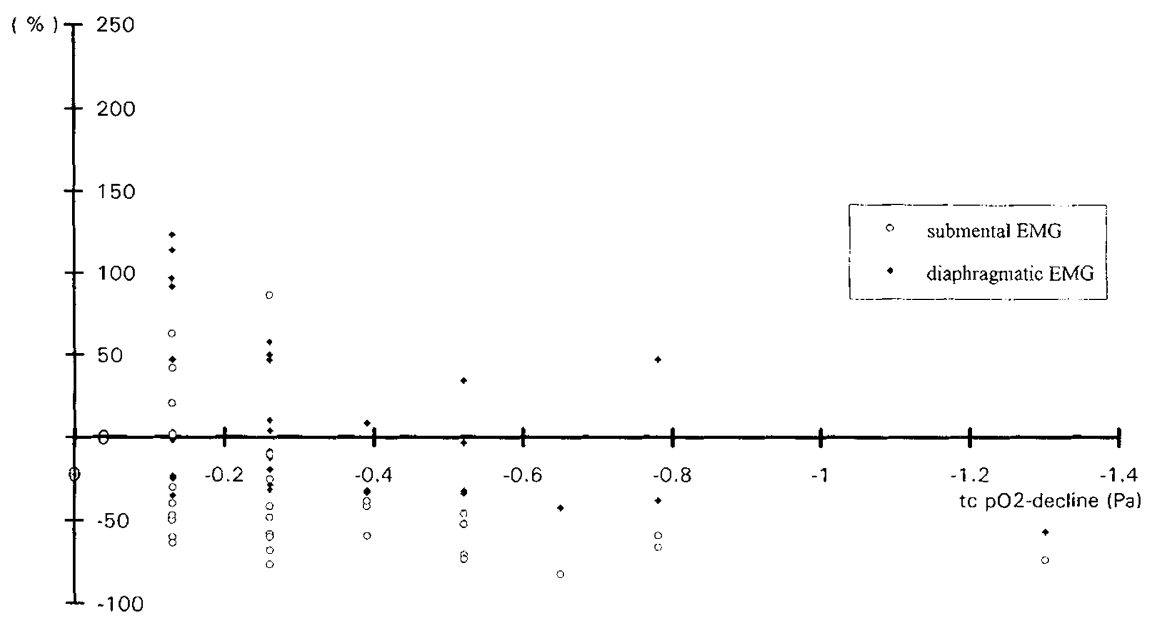

Figure 3. Change of submental and diaphragmatic EMG activity in relation to tcpO $\mathrm{O}_{2}$ decline during N-REM sleep apneas (\%). Data show a Spearman rank correlation $p<0.05$. 


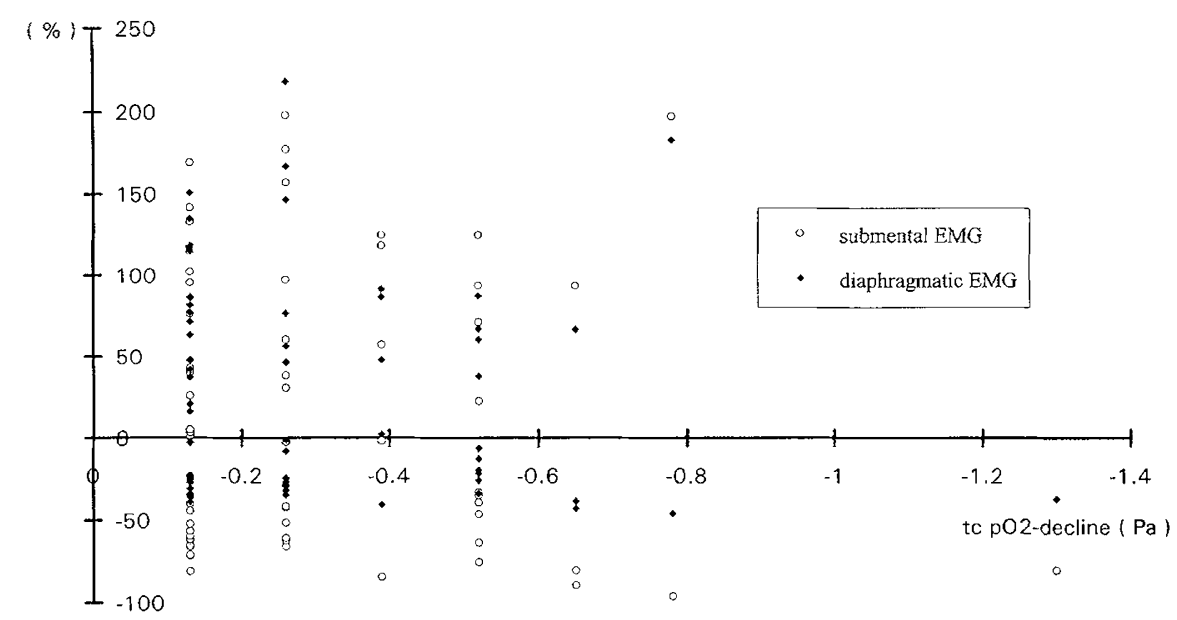

Figure 4. Change of submental and diaphragmatic EMG activity in relation to tcpO $\mathrm{O}_{2}$ decline during REM sleep apneas (\%).

Table 4. Number of apneas occurring with or without a phasic, simultaneous EMGsub and EMGdia activation at resolution

\begin{tabular}{|c|c|c|c|c|c|}
\hline REM:* & & & N-REM: & & \\
\hline With & 13 & 31 & With & 8 & 18 \\
\hline Withou & 16 & 9 & Without & 4 & 3 \\
\hline tcpO $\mathrm{O}_{2}$-decline $(\mathrm{Pa})$ & $0-0.13$ & $0.26-1.30$ & & $0-0.13$ & $0.26-1.30$ \\
\hline
\end{tabular}

* Fisher's exact test: $p<0.01$.

airways (8), an augmented genioglossus EMG activity during periods of sleep-related partial airway obstruction has been described in children (29), which was significantly correlated with tc $\mathrm{pO}_{2}$ decrease. A certain but not significant decrease with no relation to $t c \mathrm{pO}_{2}$ levels was found in five of seven children with obstructive apneas mainly due to enlarged tonsils (9). Using two surface electrodes in the anterior sublingual position no distinct sublingual EMG activity changes could be measured during apneas (27). A correlation to tc $\mathrm{pO}_{2}$ levels was not included in that study. The increase of EMGsub accompanying the onset of apneas as found in the present study could be part of a compensating mechanism to counteract instability of the pharyngeal walls, perhaps due to an insufficient activity of other muscles in the upper pharyngeal region (m. stylopharyngeus, $m$. palatopharyngeus, $m$. pharyngeus constrictor), such as has been shown in adults (30) or at the laryngeal level as shown in preterm infants (31). In the present study the increased submental EMG activity at the onset of some apneas was evoked by a deep sigh followed by an inactive apnea. In these cases the onset of breathing activity revealed an obstruction of the upper airways, turning the event into an apnea of the "inactive-obstructive" typc. The negative pressure within the upper airways during the sigh at apnea onset possibly leads to obstruction that is first noticeable at the recurrence of breathing (Figs. 1, 2, and 5). Such a process has been described using endoscopy (31). Thus the increase of submental EMG activity could neither prevent nor immediately overcome upper airway obstruction in preterm infants, possibly due to adhesive forces causing obstruction in the region of the pharyngeal walls.

Submental and diaphragmatic EMG during apneas. In contrast to the EMGsub activation at onset of apneas, submental EMG activity showed a decrease during REM sleep in about $51 \%$ of the events and in N-REM sleep in approximately $85 \%$,
Table 5. Distribution of apneas with (+) and without (-) occurrence of bradycardia in relation to simultaneous presence of submental and diaphragmatic EMG decrease during apneas

\begin{tabular}{|c|c|c|c|c|}
\hline \multirow[b]{3}{*}{ EMGsub + dia } & \multicolumn{4}{|c|}{$\begin{array}{c}\text { No. of apneas with }(+) \text { and without }(-) \\
\text { bradycardia }\end{array}$} \\
\hline & \multicolumn{2}{|c|}{$\mathrm{REM}^{*}$} & \multicolumn{2}{|c|}{$\mathrm{N}-\mathrm{REM}+$} \\
\hline & + & - & + & - \\
\hline Decrease & 8 & 10 & 10 & 4 \\
\hline No decrease & 1 & 50 & 3 & 16 \\
\hline
\end{tabular}

which correlated with the decline of tcpO $\mathrm{O}_{2}$ during the events: tcpO $\mathrm{O}_{2}$-levels decreased with submental EMG activity (Fig. 3, $p<0.05)$. These findings are in contrast with observations of infants at a mean age of 46 mo (9), showing a preferential increase of submental EMG during the course of obstructive sleep apneas which were correlated with $t c \mathrm{pO}_{2}$. A decreased ventilatory responsiveness to hypoxia has been described in preterm infants (32) as well as in "near-miss victims" of SIDS (33). Thus a predominant decrease in submental EMG duting apneas in N-REM sleep could point toward a progressing instability of upper airway maintenance. The correlation between decreasing EMGsub and falling tcpO $\mathrm{O}_{2}$ levels could be related to decreasing central drive during the events, especially in that sleep phase. A decline of inspiratory diaphragmatic EMG activity has been found in preterm infants (27), possibly reflecting a decreased central drive influenced by an intercostal-phrenic inhibition due to mechanoreceptors (34). Although increased tcpCO $\mathrm{CO}_{2}$ levels, which are closely connected with a decrease of tcpO $\mathrm{O}_{2}$ during apneas, can have an effect on ventilatory response (14), we did not find a correlation between tcpCO $\mathrm{CO}_{2}$ and $\mathrm{EMG}$ activity. The elevation of tcpCO $\mathrm{CO}_{2}$ levels by transcutaneous measurement in our study was not higher than $0.27 \mathrm{~Pa}$ possibly due to the brief duration of the apneas observed, which did not exceed $22 \mathrm{~s}$.

Submental and diaphragmatic EMG at termination of apneas. Recovery of upper airway maintenance seemed less dependent on the continuity of submental muscle force during the obstructive event alone and more on the "reflex-like" 


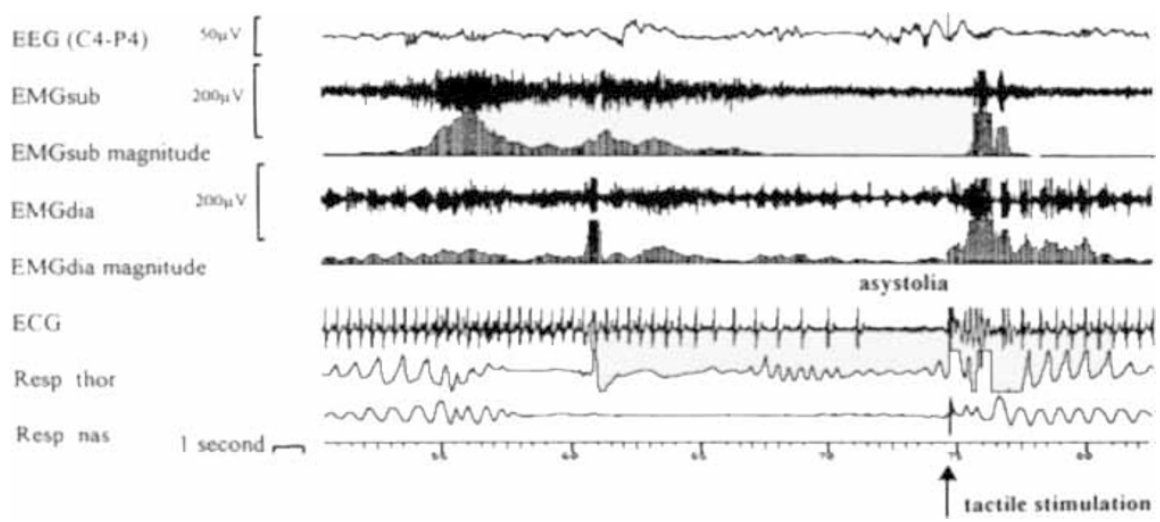

Figure 5. Illustration of an apnea with asystolia. During one of a number of mixed apneas recorded in this infant, bradycardia, which coincides with onset of breathing movements against obstruction, progressed to asystolia, whereas tcp $\mathrm{O}_{2}$ dropped from 5.5 to $5.1 \mathrm{~Pa}$ (42 to $38 \mathrm{~mm} \mathrm{Hg}$ ). A slight tactile stimulation evoked the missed parallel activation of EMGsub and EMGdia and led to immediate recovery of both cardiorespiratory and respiratory functions, remarkably without change of the sleep phase (EEG arousal).

appearance, similar to a gasp, of a combination of short expiration and deep inspiration apparently triggered by the decline of $\mathrm{tcpO}_{2}$ in REM sleep. An isolated increase of EMGsub was observed in several mixed and obstructive apneas without reestablishing upper airway patency (Fig. 2). This observation emphasizes the relevance of the simultaneous appearance of both EMGsub and EMGdia activation in resolution of apneas. A deep inspiration during an obstructive apnea alone might not overcome the adhesive force of the narrowed pharyngeal sidewalls and the tongue and could intensify this effect through a negative inspiratory pressure within the upper airways. Thus the observed expiratory effort prior the gasp could terminate the obstruction due to a positive airway pressure. The described simultaneous activation of both muscle groups requires a well developed integration of afferent and efferent neuronal pathways. Gasps are generated by neuronal pathways different from normal breathing activity in cats (15). Moreover, genioglossus EMG activity has been found to be more dependent on peripheral chemoreceptors, whereas the diaphragm seems to be preferentially controlled by central chemoreceptors (35). Gasping, in relation to apnea and hypoxia as an autoresuscitating mechanism, has been described in animals $(17,36$, 37) as well as in infants (38). An age-related autoresuscitating gasping mechanism could be demonstrated in young and adult mice with a failure in gasping during a period of time in the maturing mice (16). The occurrence of an autoresuscitating gasp in spontaneous recovery from hypoxic apneas could be suppressed by low $\mathrm{PO}_{2}$ levels in adult and infant mice (17). The significant correlation of the incidence of the apnea terminating simultaneous EMG activation of both EMGsub and EMGdia points to the important role of chemoreceptors in this apnea terminating mechanism in REM sleep. In N-REM sleep we found that this EMG pattern of apnea termination did not correlate with $\mathrm{tcpO}_{2}$ levels; it predominated independent of the degree of the decline of $t \mathrm{cpO}_{2}$. This could point to other mechanisms possibly involved in activation of EMGsub and EMGdia in terminating apneas observed in trachetomized lambs (39). The gasping mechanism we observed could not be considered unreservedly as a part of an "arousal reaction" because it was not related to a change of sleep phase, which includes an "EEG arousal," connected with a change in EEG activity or an awakening $(23,24)$. EEG arousal accompanying recovery from airway obstruction induced by neck flexion during sleep has been found in healthy newborns (40), the missing EEG arousal being related to a delayed and incomplete reopening of upper airways. This is in contrast to our findings of a complete recovery of upper airway patency as well as cardiac functions without a change in sleep phase. Arousal response to hypoxia has been found to be diminished in near-miss SIDS infants versus healthy infants $(14,32)$ as well as in infants with bronchopulmonary dysplasia (25). It has been hypothesized that a failure in arousal or gasping might be the decisive failure in SIDS victims if some deficit in cardiorespiratory, chemoreceptor, or temperature control is also present (18).

Bradycardia and asystolia in mixed and obstructive apneas. Incidence of bradycardia in our study was, with two exceptions, highly related to mixed and obstructive apneas. The distinct correlation between the combined decrease of EMG activities of both muscle groups, and the incidence of bradycardia points to an interaction of different neuronal pathways within the CNS, including the brainstem. A significant sudden decrease of EEG activity just at onset of inactive apneas in REM sleep as well as mixed and obstructive apneas in N-REM sleep (41) could be the consequence of a sudden decrease of central sympathetic activity. If this change were related to a lost balance between sympathetic and vagal activity, a consecutive increase of vagal tonus could facilitate the occurrence of bradycardia, more likely by intrathoratic pressure changes (10) than by hypoxia (42), which we did not find during apnea. An increased vagal tonus has been presumed by increased incidence of severe bradycardia and asystolia due to ocular compression (43) found in infants at risk of SIDS. On the other hand, decreased heart rate could also be considered as a protecting mechanism in reducing oxygen consumption during apnea (44), and not as reflecting a dysfunction. Such a protective mechanism could even be additive in provocation of bradycardia. In all of our observed apneas the gasping mechanism was not only followed by a reestablished upper airway patency but also by the termination of bradycardia: a "cardio- 
respiratory arousal." This cardiorespiratory arousal seems to be in contrast to arousal responses, including EEG arousal by changing sleep phase, which have been found to be abnormal in near-miss SIDS infants (14). The dependency of gasping on the degree of $t \mathrm{cpO}_{2}$ decline, which we found to be significant in REM sleep, points out the important role of chemosensitivity in triggering this mechanism. A tcpO fall below a critical minimal level may lead to a suppression of this cardiorespiratory arousal, thus issuing a life-threatening event.

Acknowledgment. We thank Dr. Jarold Knispel for his help in preparing the manuscript.

\section{REFERENCES}

1. Hoppenbrouwers T, Hodgman JE, Harper RM 1977 Polygraphic studies of normal infants during the first six months of life. MII. Incidence of apnea and periodic breathing. Pediatrics 60:418-425

2. Rigatto H, Brady JP 1972 Periodic breathing and apnea in preterm infants. II. Hypoxia as a primary event. Pediatrics 50:219-228

3. Albani M, Bentele KHP, Budde C, Schulte FJ 1985 Infant sleep apnea profile: Preterm versus term infants. Pediatrics 143:261-268

4. Bentele KHP, Albani M, Budde C, Schulte FJ 1985 Sleep apnea profile in preterm infants recovering from respiratory distress syndrome. Arch Dis Child 60:547--554

5. Mathew OP, Roberts JL, Thach BT 1982 Pharyngeal airway obstruction in preterm infants during mixed and obstructive apnea. J Pediatr 100:964-968

6. Thach BT 1983 Pharyngeal airway obstruction in infantile apneic spells. In: Tildon JT, Roeder LM, Steinschneider A (eds) Sudden Infant Death Syndrome. Academic Press, New York, p 286

7. Tonkin SL 1983 Pharyngeal airway obstruction: Signs, factors in its production. In Tildon JT, Roeder LM, Steinschneider A (eds) Sudden Infant Death Syndrome. Academic Press, New York, pp 453-465

8. Remmers JE, De Groot WT, Sauerland WK 1978 Pathogenesis of upper airway occlusion during sleep. J Appl Physiol 44:931-938

9. Praud JP, D'Allest AM, Delaperche S, Bobin S, Gaultier C 1988 Diaphragmatic and genioglossus electromyographic activity at the onset and at the end of obstructive apnea in children with obstructive sleep apnea syndrome. Pediatr Res 23:1-4

10. Guillcminault C 1988 SIDS, near miss SIDS and cardiac arrhythmia. NY Acad Sci 533:359-367

11. Wulbrand H., Bentele KHP, Albani M., Schulte FJ 1987 Patterns of submental and diaphragmatic muscle activity during mixed and obstructive apnea in infants recovering from bronchopulmonary dysplasia. Pediatr Res 22:222(abstr)

12. Guilleminault C, Ariagno R, Korobkin R, Nagel L, Baldwin R, Owen M 1979 Obstructive sleep apnea and near miss for SIDS.: I. Report of an infant with sudden death. Pediatrics 63:837-843

13. Schulte FJ, Albani M., Schnitzer H., Bentele KHP 1982 Neuronal control of neonatal respiration-slecp apnea and the sudden infant death syndrome. Neuropediatrics $13: 3-14$

14. Hunt CE 1981 Abnormal hypercarbic and hypoxic sleep arousal responses in nearmiss SIDS infants. Pediatr Res 15:1462-1464

15. St. John WM 1990 Neurogenesis, control, and functional significance of gasping. J Appl Physiol 50:984

16. Jacobi MS, Thach BT 1989 Effect of maturation on spontaneous rccovery from hypoxic apnea by gasping. J Appl Physiol 66:2384-2390

17. Gershan WM, Jacobi MS, Thach BT 1990 Maturation of cardiorespiratory interactions in spontaneous recovery from hypoxic apnea (autoresuscitation). Pediatr Res $28: 87-93$
18. Hunt CE 1992 The cardiorespiratory control hypothesis for sudden infant death syndrome. Clin Perinatol 19:757-771

19. Calder N, Williams B, Smyth J, Boon A, Kumar P, Hanson M 1994 Absence of ventilatory responses of mild hypoxia and air in infants who have had bronchopulmonary dysplasia: Implications for the risk of sudden infant death. Pediatr Res 35:677-681

20. Werthammer J, Brown E, Ncff RK, Tauesch HW 1982 Sudden infant death Syndrome in infants with bronchopulmonary dysplasia. Pediatrics 69:301-403

21. Parmelee AH, Schulte FJ, Akiyama Y 1968 Maturation of EEG activity during sleep in premature infants. Electroenceph Clin Neurophysiol 24:319-329

22. Schulte FJ, Michaclis R, Noite R, Albert R, Parl U, Lasson U 1969 Brain and behavioural maturation in newborn infants of diabetic mothers. I. Nerve conduction and EEG pattern. Neuropaediatrie 4:349-460

23. Anders T, Emde R, Parmelee A 1971 A manual of Standardized Terminology, Technology, and Criteria for Scoring of States of Sleep and Wakefulness in Newborn Infants. UCLA Brain Information Scrvice/BRI Publications Office, Los Angeles

24. Coons S, Guilleminault C 1985 Motility and arousal in near miss sudden infants death syndrome. J Pediatr 107:728-732

25. Garg M, Kurzner DI, Bautista DB, Keens T 1988 Hypoxic arousal response in infants with bronchopulmonary dysplasia. Pediatrics 82:59-63

26. McCulloch K, Brouillette RT, Guzzetta AJ 1982. Arousal responses in near-miss sudden infant death syndrome and in normal infants. J Pediatr 101:911-917

27. Gauda EB, Miller MJ, Carlo W, DiFiore JM, Martin R 1989 Genioglossus and diaphragm activity during obstructive apnea and airway occlusion in infants. Pediat Res 26:583-587

28. Pocts C, Southall P 1994 Noninvasive monitoring in infants and children: Practical considerations and areas of concern. Pediatrics 93:737-746

29. Jeffries B, Brouillette, Hunt C 1984 Electromyographic study of some accessory muscles of respiration in children with obstructive sleep apnea. Am Rev Respir Dis 1129:696-702

30. Guilleminault C, Ariagno R, Korokbin R, Nagel L, Baldwin R, Owen M 1978 Obstructive slecp apnea: Electromyographic and fiberoptic studies. Exp Neurophysiol 21:299-306

31. Ruggins NR, Milner AD 1993 Site of upper airway obstruction in infants following an acute life-threatening event. Pediatrics 91:595-601

32. Rigatto H, Brady JP, Verduzco R, de la Toorc 1975 Chcmoreceptor reflexes in preterm infants. $\mathrm{I}$. The effect of gestational and postnatal age on the ventilatory response to inhalation of $100 \%$ and $15 \%$ oxygen. Pediatrics 55:202-218

33. Hunt $\mathrm{CE}$, Mc Culloch K, Brouillette RT 1981 Diminished hypoxic ventilatory responses in near-miss sudden infant death syndrome. J Appl Physiol 10:1313-1317

34. Chernick V 1981 The Fetus and the Newborn. In: Hornbein TF (ed) Regulation of Breathing, Vol. II. Marcel Dekker, New York, pp 1142-1179

35. Bruce EN, Mitr J, Cherniak N 1982 Central and peripheral chemoreceptor inputs to phrenic and hypoglossal motoneurons. J Appl Physiol Respir Environ Exercise Physiol 53:1504-1511

36. St.Ambrigo J, Milic-Emily J, Camporesi E 1971 Occurence of a deep breath after a period of airway occlusion. Pflügers Archiv 327:95-104

37. Guntheroth WG, Kawabori I 1975 Hypoxic apnea and gasping. J Clin Invest 56:1371

38. Stevens LH 1965 Sudden unexplained death in infancy. Am J Dis Child 110:243-247

39. Baker S., Fewell JE 1987 Effects of hyperoxia on the arousal response to upper airway obstruction in lambs. Pcdiatr Res 21:116-120

40. Stark A, Thach B 1981 Recovery of airway patency after obstruction in normal infants. Am Rev Respir Dis 123:691-693

41. Wulbrand $\mathrm{H}$, v.Zezschwitz HG, Mende W, Schuite FJ 1991 EEG and submental EMG activity during apneas in preterm infants. J Perinat Med 19:263(abstr)

42. Kelly DH, Shannon DC 1991 Sudden severe bradycardia in infancy. Pediatr Pulmono 10:199-204

43. Kahn A, Riazi J, Blum D 1983 Ocular cardiac reflex in near miss for sudden infant death syndrome infants. Pediatrics 71:49-52

44. Shepard JT 1986 Cardiac Mechanoreceptors. In: Fozzard HA, Haber E, Jennings RB, Katz AM (eds) The Heart and Cardiovascular Sy'stem. Raven Press, New York, pp 1535-1558 